\title{
TERAPIA OCUPACIONAL Y ANDRAGOGÍA. UN LLAMADO A LA INCLUSIÓN DESDE EL FIN DEL MUNDO
}

OCCUPATIONAL THERAPY AND ANDRAGOGY. AN INVITATION TO THE INCLUSION FROM THE END OF THE WORLD

\section{CRISTIAN ARANDA F. ${ }^{1}$, ANDREA YUPANQUI C. ${ }^{2}$, WILSON VERDUGO H. ${ }^{3}$}

\section{Resumen}

Se presenta un estudio cuantitativo destinado a determinar el interés por estudiar de adultos mayores institucionalizados de la ciudad de Punta Arenas, junto con la identificación de factores favorecedores y obstaculizadores. Se incluyó a 18 adultos mayores institucionalizados residentes del Establecimiento de Larga Estadía del Adulto Mayor de Punta Arenas. Se obtiene que el $80 \%$ de los adultos mayores encuestados reportan un alto interés por estudiar, con particular énfasis en las mujeres. Se encontró una correlación positiva: a mejor constitución de red social de apoyo (familia e institución de residencia), mayor interés por estudiar. La mayor parte quisiera terminar sus estudios inconclusos, y manifiestan interés en estudiar historia, idiomas e incluso, estudios universitarios. Los resultados de esta investigación invitan a reflexionar sobre la imagen negativa que tiene la sociedad acerca de esta etapa de la vida, sobre la necesidad de cambio para poder ofrecer los espacios necesarios para seguir desarrollándose, especialmente en el ámbito educativo, donde la andragogía es esencial para garantizar la participación, inclusión y contribución de los adultos mayores en la sociedad. Aquí la Terapia Ocupacional tiene una responsabilidad social trascendental.

\section{Palabras clave:}

Adultos Mayores, Andragogía, Inclusión, Terapia Ocupacional.

\footnotetext{
${ }^{1}$ Terapeuta Ocupacional, Magíster en Educación mención currículo, Profesor Asistente Depto. de Terapia Ocupacional, Universidad de Magallanes. Av. Bulnes № 01855, fono contacto 5661 207924, cristian.aranda@umag.cl

2 Terapeuta Ocupacional, Magíster en Educación mención currículo, Profesor Asistente Depto. de Terapia Ocupacional, Universidad de Magallanes. Av. Bulnes № 01855, fono contacto 5661 207924, andrea.yupanqui@umag.cl

3 Terapeuta Ocupacional, Magíster (C) en Educación mención currículo, Profesor Asistente Depto. de Terapia Ocupacional, Universidad de Magallanes. Av. Bulnes № 01855, fono contacto 5661 207924, wilson.verdugo@umag.cl
} 


\begin{abstract}
Quantitative study designed to determine the interest in studying the institutionalized older people in Punta Arenas city, along with the identification of factors favoring and hindering. The sample included 18 institutionalized elders residents of long-stay Facility for the Elderly of Punta Arenas. The 80\% of older people report a high interest in the study, with particular emphasis on women. It founds a positive correlation: a better incorporation of social support network (family and institution of residence), greater interest in studying. Most want to finish their studies unfinished, and expressed interest in studying history, languages and even higher education. The results of this research invite to reflection on the negative image that society has about this stage of life, about the need for change in order to provide the necessary space for further development, especially in education, which is essential andragogy to ensure participation, inclusion and contribution of older people in society. Here the Occupational Therapy has a crucial social responsibility.
\end{abstract}

Key words:

Older People, Andragogy, Inclusion, Occupational Therapy.

\section{INTRODUCCIÓN}

En la búsqueda de relacionar y orquestar dos temas de interés en la actualidad, como lo es la educación y el exponencial aumento de la sobrevida de las personas en nuestro país y a nivel mundial, es que el tema de la andragogía aparece como un concepto interesante a analizar. Para contextualizar la presente investigación, se hará una revisión sobre el aumento explosivo de la población mayor de 60 años en todo el mundo, el concepto de educación, la educación pensada para adultos y una reflexión acerca de la necesidad del cambio e innovación en la andragogía.

\section{Proceso de Envejecimiento Poblacional}

El envejecimiento poblacional es definido como el aumento de la proporción de personas de edad avanzada (60 años y más) con respecto a la población total (Kornfeld et al., 2006, p. 4). Esto se ha visto con mayor énfasis durante las últimas décadas a nivel mundial, donde la población ha aumentado en forma considerable la cantidad de personas de 60 años y más, sobre todo en países en vías de desarrollo.

Latinoamérica no ha sido la excepción y las personas de edad han adquirido una mayor representación numérica. Así lo refiere la Comisión Económica Para América Latina y el Caribe (CEPAL), demostrado en las siguientes proyecciones: “En el año 2000, la población regional de 60 años y más era de 43 millones de personas, cifra que irá en aumento hacia el año 2025, cuando las personas de edad alcancen los 100,5 millones. Para 2050, en tanto, la población latinoamericana y caribeña de esta edad podría llegar a los 183,7 millones, superando ampliamente las cifras observadas hacia fines del siglo anterior" (CEPAL, 2009, p.13).

Según datos entregados por la Organización de las Naciones Unidas (ONU), la proyección 
hacia el año 2025, es que la población mundial (de todas las edades) se multiplicará por tres, las personas mayores de 60 años lo hará en 6 veces y la población mayor de 80 años lo hará en diez veces. En estos momentos, la población de personas mayores de 60 años aumenta en un número no inferior a 1.000 .000 por día a nivel mundial. Otro dato interesante es que el $80 \%$ de los adultos mayores vive en países en vías de desarrollo como el nuestro. Actualmente el $11 \%$ de la población chilena tiene más de 60 años (Salgado et al., 2002, p.15).

A estos datos agregamos que nuestra región de Magallanes (ciudad de Punta Arenas, ubicada al extremo sur de Chile y del continente americano), en el último censo del año 2003 tenía una población de 150.826 habitantes, de los cuales cerca de 17.026 personas tenían más de 60 años, lo que equivale al $11,3 \%$ de la población. (INE, 2003, p. 18).

¿Y a qué se debe este envejecimiento de la población en Chile y en el mundo? Según Kornfeld, R. y cols. (2004), este cambio se produce básicamente debido a dos factores: a la transición demográfica y a la transición epidemiológica.

La transición demográfica se refiere a los cambios en la estructura de la población, principalmente por la baja en las tasas de fecundidad y también de mortalidad, es el cambio en el dibujo de la población en general que hace 20 años era un triángulo con una base muy amplia (indicando el gran número de recién nacidos) que se iba enangostando progresivamente hacia arriba. Actualmente ese dibujo parece más un rectángulo que un triángulo con una base ancha y una parte superior cada vez más parecida. La proyección es que desde el año 2025 hacia el futuro este dibujo volverá a parecer una pirámide, pero invertida con base angosta y una parte superior ancha. La transición epidemiológica es producto de la disminución de patologías que provocaban la muerte en adultos mayores y que actualmente, gracias al avance de la tecnología farmacéutica y de conocimientos médicos, la sobrevida ha aumentado ostensiblemente en todo el mundo.

\section{Educación}

Para la Organización de las Naciones Unidas para la Educación, Ciencia y la Cultura (UNESCO), la educación es un derecho humano y pilar central para alcanzar y proteger el resto de derechos de los seres humanos. Tiene un fin instrumental, puesto que su objetivo último es mejorar las condiciones de vida de las personas, en los ámbitos de salud, libertad, seguridad, bienestar económico, participación social y política. Además, la considera como un proceso permanente, centrado en la humanización, elemento que valida a la persona en la interacción con sus semejantes: “Aprender a Ser, para que florezca mejor la propia personalidad y se esté en condiciones de obrar con creciente capacidad de autonomía, de juicio y de responsabilidad personal. Con tal fin, no menospreciar en la educación ninguna de las posibilidades de cada individuo: memoria, razonamiento, sentido estético, capacidades físicas, aptitud para comunicar" (Delors, 1996, p. 34). 
En este sentido, el año 2000 los representantes de más de 160 gobiernos se reunieron en el Foro Mundial sobre la Educación en la ciudad de Dakar (África), donde se estableció un Marco de Acción con 6 objetivos. Bajo el concepto "Educación para todos" (EPT), la comunidad internacional se comprometió a alcanzar este objetivo para el año 2015. En este contexto, algunos de sus objetivos están referidos a "atender las necesidades de aprendizaje de los jóvenes y adultos a lo largo de toda la vida" y "alfabetización de los adultos", todo enmarcado en el mejoramiento de la calidad de la educación y en el compromiso de promover las oportunidades de un acceso equitativo e inclusivo a la educación para todos los ciudadanos del mundo (UNESCO, 2009, p. 6).

En nuestro país, la Constitución Política establece que "la educación tiene por objeto el pleno desarrollo de la persona en todas las etapas de su vida (...), corresponderá al Estado, asimismo, fomentar el desarrollo de la educación en todos sus niveles" (Art. 19.10).

En resumen, tanto a nivel internacional como nacional, la educación es esencialmente reconocida como un derecho, vigente durante toda la vida de los seres humanos, caracterizado por el fomento del desarrollo integral de los individuos, lo que se encuentra vinculado al contexto históricosocial en el que se esté inmerso.

\section{Educación para Adultos}

La educación para adultos ha sido denominada como "andragogía", proviene de dos raíces griegas: "andros", que significa hombre y "gogos", guiar o conducir (Blake, 2008, p. 190).

El término "andragogik" fue utilizado por primera vez en 1833 por el filósofo alemán Alexander. Más adelante, Malcolm Knowles, retomó y amplió este concepto en 1970 en su obra "The adult learner: a neglected species" (Rumbo, 2006, p. 626). Una de las definiciones más actuales de andragogía es la siguiente: "es la ciencia y el arte que, siendo parte de la antropología y estando inmersa en la educación permanente, se desarrolla a través de una praxis fundamentada en los principios de participación y horizontalidad; cuyo proceso, al ser orientado con características sinérgicas por el facilitador del aprendizaje, permite incrementar el pensamiento, la autogestión, la calidad de vida y la creatividad del participante adulto, con el propósito de proporcionarle una oportunidad para que logre su autorealización" (Alcalá, 2001, p. 6)

La intención de poner énfasis en el concepto de andragogía y no en la gerontología es justamente porque la primera incluye a la segunda y un trabajo que tiene como objetivo la inclusión no podría excluir a aquellos adultos que invariablemente se dirigen a esta etapa de la vida, quienes debiesen prepararse para vivenciar de la mejor forma posible la etapa que está por venir. 
Necesidad de Innovación y Cambio en

Andragogía

Los avances en materia educativa muchas veces son lentos y resistentes al cambio. Así lo reflejan los avances hacia la consecución de los objetivos de la UNESCO, los que se han visto obstaculizados por diversos factores, entre ellos el fracaso de los gobiernos en sus tentativas de acabar con las persistentes desigualdades basadas en los ingresos, el sexo, el lugar de domicilio, la pertenencia étnica, la discapacidad y otros factores de desventajas. A no ser que los gobiernos actúen para reducir las disparidades mediante reformas eficaces de sus políticas, las promesas de lograr dichos objetivos no se cumplirán (UNESCO, 2009, p. 1). Líderes dispuestos a cambiar las políticas públicas, de democratizar el acceso a los distintos servicios, podrán lograr disminuir las desigualdades sociales en todos los ámbitos de participación social a nivel nacional.

Este aumento en las expectativas de sobrevida de los seres humanos trae consigo múltiples desafíos. La apuesta de los autores de este estudio se proyecta hacia un cambio cultural, orientado hacia la idea que no sólo el derecho a la vida es inviolable, sino también el derecho a la buena vida, y en tanto tal, se extiende a todas las circunstancias de la existencia, a los derechos y libertades inalienables del hombre.

Una opción por la vida es el estudio sistemático por seguir aumentando las expectativas de vida de los seres humanos, y la opción por la buena vida es considerar los ambientes que son factibles de crear por la comunidad y sus líderes que favorezcan el crecimiento y pleno desarrollo del ser humano en su dimensión social, donde la educación es esencial en la integración de las personas a ese ambiente social, a través de la educación conseguimos hacernos parte de los avances de la misma sociedad y no quedarnos rezagados (o abandonados) por el paso del tiempo.

Particularmente nuestro país se caracteriza por considerar al adulto mayor como un ser frágil, poco participativo y de escasa utilidad para la sociedad, ya que no puede entregar productos ni contribuir al progreso social. Los prejuicios sociales negativos son parte del imaginario social, por lo tanto, se hace necesario favorecer la convivencia digna y el desarrollo de los adultos mayores, cambiar esa noción por la idea de que estos hombres y mujeres, con la sapiencia que dan los años y la fuerza que proporcionan las experiencias pasadas, con la decidida voluntad de enfrentar los cambios, deben participar de procesos educativos desarrollados y adecuados a sus propias características (Barros, 1996).

Asimismo, la investigación ha demostrado que la educación es uno de los predictores más fuertes de la mantención de la función cognitiva (Rowe y Kahn, 1998; Schaie, 1994), puesto que los adultos mayores también necesitan habilidades para hacer frente a las preocupaciones relacionadas con la edad, tales como el ocio, la jubilación, la salud, la muerte, la vivienda y las finanzas (Fisher y Wolf, 1998). Aumentar los conocimientos de los adultos mayores y el rendimiento de actividades de bienestar 0 de temas económicos, por ejemplo, permite reducir la 
dependencia de los programas de bienestar social y mejora la calidad de vida.

A pesar de las ventajas físicas, mentales y psicológicas de la participar en experiencias educativas, los adultos mayores participan en programas educativos a un ritmo mucho menor que la población adulta en general, y Chile no es la excepción.

El incluir a los adultos mayores en los procesos educativos es parte importante de los principios democráticos en que la sociedad se sustenta, por lo tanto, es necesario concebir la necesidad del cambio de paradigma, por uno en el cual la sociedad sea inclusiva en todos los ámbitos.

En este sentido, y considerando los elementos antes expuestos, el presente estudio se propuso el objetivo de determinar el interés por estudiar manifestado por adultos mayores institucionalizados de la ciudad de Punta Arenas, junto con identificar sus factores favorecedores y obstaculizadores.

\section{METODOLOGÍA}

La metodología empleada en este estudio fue de carácter cuantitativo, de tipo causal, cuyo diseño fue no experimental, transversalcorrelacional.

Esta investigación corresponde a un estudio de caso único y situacional centrado en el marco geográfico de la región de Magallanes, comuna de Punta Arenas. El grupo investigado reside en el Establecimiento de Larga Estadía del Adulto Mayor de Punta Arenas.
Este estudio aseguró la confidencialidad a todos quienes participaron voluntariamente, mediante un consentimiento informado, según el cual la información obtenida sería de uso exclusivamente académico y los nombres de los participantes se mantendrían en el anonimato.

Se aplicó una encuesta pre-diseñada elaborada por los investigadores para la búsqueda de factores que influyen en el interés de adultos mayores en estudiar. El instrumento consideró las variables de género, edad, tiempo de institucionalización, nivel de estudios previos, estado civil, red social y áreas de interés.

Se utilizó un muestreo de tipo no probabilístico de tipo intencional, cuyos criterios seleccionados fueron: ser adulto mayor perteneciente al Establecimiento de Larga Estadía del Adulto Mayor de Punta Arenas; presentar facultades mentales indemnes según se indique en ficha clínica; evidenciar posibilidad de comunicación oral o escrita.

Se analizaron 18 encuestas de un total de 22 adultos mayores institucionalizados. $\mathrm{El}$ análisis de la información se basó en la correlación estadística que presentaron las distribuciones de frecuencias de las variables sobre las cuales existen relaciones de determinación.

\section{RESULTADOS}

Los resultados permiten sugerir que existe una correlación positiva entre red de apoyo e interés por estudiar: a mejor constitución de red social de apoyo (familia e institución de 
residencia), mayor interés por estudiar en adultos mayores institucionalizados de la ciudad de Punta Arenas. Ellos manifiestan un marcado interés por estudiar $(80 \%$ de los encuestados). Un porcentaje importante de ellos tiene bajo nivel de enseñanza (70\% educación básica incompleta), edad promedio 74 años, estado civil viudo y menos de 7 años de institucionalización. Los elementos que aparecen como factores que no favorecen el interés por estudiar son presentar el diagnóstico de depresión y una red social precaria y disfuncional.

Un interesante hallazgo apunta a que las mujeres manifiestan mayor interés que los hombres por estudiar (el 100\% de las mujeres manifiesta algún interés por estudiar, mientras que sólo el $57 \%$ de los hombres lo expresa). Actualmente, ninguno de los adultos mayores participantes del estudio realiza alguna actividad relacionada al estudio, y lo más preocupante, todos los encuestados expresan que desconocen cualquier oportunidad de poder hacerlo. Declaran que si tuviesen la oportunidad de estudiar, la mayor parte de los encuestados $(60 \%)$ quisiera terminar sus estudios inconclusos.

Por último, refieren que otras áreas de interés por estudiar serían la historia, idiomas e incluso, estudios universitarios.

\section{DISCUSIÓN}

Los hallazgos obtenidos podrían evidenciar la importante influencia que ha tenido la intervención de Terapia Ocupacional en estos adultos mayores durante los últimos 6 años en el servicio, donde se ha instalado la necesidad de ocupaciones significativas como fomento de la salud, bienestar, calidad de vida y por sobre todo, de esperanza, elemento que nos obliga a continuar luchando por una sociedad más inclusiva.

En definitiva, este trabajo pretende hacer un llamado urgente para que los Terapeutas Ocupacionales asumamos la responsabilidad social que tenemos con los adultos mayores de nuestro país, considerando los siguientes elementos:

- Lograr la participación plena de los adultos mayores a través del desarrollo de planes educativos que garanticen el adecuado manejo de sus características. Para ello es necesario asumir que es necesario modificar el imaginario social a través de la participación de distintos agentes de cambio, de distintas disciplinas, que fomenten con su participación en la planificación, organización, conducción e implementación del trabajo colectivo, que implique un cambio a gran escala y que logre la inclusión de los adultos mayores, que sin lugar a dudas, será beneficioso para el progreso social en general, no sólo para los adultos mayores.

- Hacernos parte como sociedad de uno de los principios directrices que regulan la convivencia de los seres humanos en nuestro planeta, como lo es la Declaración Universal de los Derechos Humanos, que en su artículo primero indica que "Todos los seres humanos nacen libres $e$ iguales en dignidad y derechos y dotados como están de razón y conciencia, deben comportarse fraternalmente los unos con los otros" (Art.1).

El hombre libre tiene derecho a desarrollarse en todas las áreas sin excepción, para ello requiere de igualdad de oportunidades para 
ingresar durante cualquier momento de su vida a cualquiera de los niveles de la educación, la sociedad actual no permite la expresión de esa necesidad en su real magnitud. Si analizamos lo que hoy sucede en nuestras sociedades, es posible ver que el hombre no suele comportarse fraternalmente los unos con los otros, lo que ocurre especialmente con aquellos que se consideran como diferentes. Por lo demás en el artículo 26 plantea que "toda persona tiene derecho a la educación".

- Avanzar hacia una educación de calidad: Hay que destacar que la educación para adultos tiene objetivos que van mucho más allá de aprender a leer o escribir. El objetivo de la educación es "el del pleno desarrollo de la personalidad humana y el fortalecimiento de respeto a los derechos humanos y a las libertades fundamentales, lo que favorecerá la comprensión, la tolerancia, y la amistad.

- Abogar por una preparación de profesionales de distintas disciplinas formados para el trabajo con adultos mayores, considerando sus características y necesidades. Las palabras de Paulo Freire, en su libro Pedagogía del Oprimido, reflexiona acerca de que "nadie educa a nadie, ni nadie aprende sólo, los hombres (mujeres) aprendemos mediatizados por el mundo" (Freire, 1975, p. 90), representa un principio fundamental al considerar una experiencia andragógica. El hecho de que cada ser humano cuenta con un potencial de experiencias dentro de su contexto socio-cultural que serán fundamentales para su aprendizaje y esto debe ser parte del docente de adultos mayores.

Para Freire la educación debe servir para que los educadores y educandos puedan conocer el mundo, leer el mundo para poder transformarlo, ¿es acaso imaginable pensar que un adulto o adulto mayor puede aún transformar el mundo?, ¿cuánto podría la sociedad en su conjunto desarrollarse a partir de ellos y ellas?

La educación por sí misma no cambia el mundo, pero sin ella es imposible hacerlo. En consecuencia, quienes participan del trabajo con adultos mayores deben tener un compromiso ético por la construcción de un mundo más inclusivo, no es posible ser indiferentes frente a las injusticias y a la discriminación, se debe mantener y promover la esperanza en el cambio. Por ello, para Freire, la realidad no es sólo el punto de partida de la educación sino también su punto de llegada. La realidad no está dada, sino dándose, la finalidad de la educación liberadora es contribuir a transformar en función de visiones de futuro que superen la existencia de opresores y oprimidos, de explotadores y explotados, de excluyentes y excluidos; es superar los obstáculos económicos, sociales, políticos y culturales que impide la realización de los seres humanos. Las prácticas educativas críticas, articuladas a praxis sociales transformadoras, hacen posible que la gente escriba su propia historia, es decir, sea capaz de superar las circunstancias y factores adversos que la condicionan (Freire, op. cit).

Después de estas consideraciones resulta pertinente preguntarse:

¿La libertad e igualdad con que nace el hombre se circunscribe a una determinada etapa etaria?, ¿El adulto mayor tiene derecho a lograr el pleno desarrollo de su personalidad?, ¿El adulto mayor puede lograr mejorar su comprensión de los fenómenos 
que ocurren en el mundo?, ¿El adulto mayor puede a través de la educación volverse más tolerante?

En síntesis, si queremos hacer de nuestro mundo un lugar más fraterno e inclusivo, se deben enfrentar los procesos de cambio sin miedos y pensando que toda la vida es un cambio dirigido hacia el crecimiento y la mejora. Y, visto desde esta perspectiva, ¿por qué no embarcarse en nuevos proyectos? La respuesta a esta pregunta debe ser contestada desde dos esferas: primero la sociedad y sus proyectos inclusivos en educación para adultos mayores, y segundo, por los mismos adultos mayores, ¿acaso no tengo más experiencia que el resto para poder vencer las vicisitudes que se presenten?

La propuesta de cambio pasa necesariamente por aunar voluntades, esas voluntades deben ser dirigidas por equipos multidisciplinarios de expertos que aporten distintas visiones del fenómeno y que comiencen con la gran tarea de cambiar el imaginario social. Una vez que este imaginario social cambie, se entregarán programas educativos orientados específicamente a los adultos mayores. De esta forma, la sociedad estará preparada para convertirse en una sociedad más participativa, más inclusiva, y más democrática.

Al ser humano no se le pueden poner restricciones basadas en prejuicios, las capacidades de los seres humanos no se pueden coartar por el hecho de llegar a una determinada edad. Si no cambiamos esta forma poco fraterna de tratarnos, seguiremos “invalidándonos" y no obteniendo los beneficios que nos entrega una parte importante de nuestra sociedad que al día de hoy aún es una minoría.

Esta situación deberá ir cambiando, entonces preparémonos para ello, imaginemos el cambio en el imaginario social, imaginemos el cambio en la planificación de los gobiernos, imaginemos a los adultos y adultos mayores aprendiendo a leer, imaginemos a los adultos mayores obteniendo títulos universitarios, entrando a estudiar postgrados, imaginemos que en el futuro no tendremos que imaginar...

\section{Ha sido establecido científicamente, que el abejorro no puede volar. \\ Su cabeza es demasiado grande y sus alas demasiado pequeñas para sostener su cuerpo. \\ Según las leyes aerodinámicas sencillamente no puede volar. ...pero nadie se lo ha dicho... ASÍ ES QUE VUELA. (Kornfeld, 2006, p. 96)}

\section{REFERENCIAS BIBLIOGRÁFICAS}

Alcalá, A. (2001). La praxis andragógica en los adultos de edad avanzada. Informe de Investigaciones Educativas, 15(1-2): 1-19.

Barros, C. (1996). Trabajando el envejecer. Cuadernos del Instituto de Sociología. Santigo: Pontificia Universidad Católica de Chile.

Blake, O. (2008). Así aprendieron a trabajar: Cómo se construyó la capacitación laboral en la Argentina. Buenos Aires: Granica S.A.

Centro Latinoamericano y Caribeño de Demografía (CELADE) - División de Población de la CEPAL. Estimaciones y proyecciones de población. [Documento en 
línea].

Disponible:

http://www.eclac.cl/celade_proyecciones/ basedatos_80.htm [Consulta: 2013, Marzo 06].

CEPAL (2009). El envejecimiento y las personas de edad. Indicadores sociodemográficos para América Latina y el Caribe. Santiago de Chile: CELADE/CEPAL.

Delors, J. (1996). La educación encierra un tesoro: Informe a la UNESCO de la Comisión Internacional sobre la Educación para el Siglo XXI. Paris: UNESCO.

Freire, P. (1975). Pedagogía del oprimido. Buenos Aires: Siglo Veintiuno.

Fullan, M. (2002). Los nuevos significados del cambio en educación. Barcelona: Octaedro.

Knowles, M. (1970). The modern practice of adult education. Andragogy versus Pedagogy. Chicago, Associations Press.

Kornfeld, R., Orellana, V., Rozowski J. y Walker, R. (2006). Teorías, Enfoques y cambios asociados al envejecimiento. Santiago de Chile: Pontifica Universidad Católica de Chile.

Ministerio Secretaría General Presidencia de la República de Chile (2002). Catastro de Población Adulta Mayor. Santiago de Chile: INE

ONU (1948, 10 de Diciembre). Declaración Universal de los Derechos Humanos.

Rowe, J.W. y Kahn, R.L. (1997). Successful aging. The Gerontologist, 37, 433-440.

Rumbo, B. (2006). La educación de las personas adultas: un ámbito de estudio e investigación. Revista de Educación, 339: 625-635.

Salgado, A., Guillén F. y Ruipérez, I. (2002). Manual de geriatría. Barcelona: Masson.

Schaie, K.W. (1994). The course of adult intelectual development. American Psychologist, 49, 304-313.

UNESCO (2009). Informe de Seguimiento de la Educación para Todos en el mundo. Superar la desigualdad: Por qué es importante la gobernanza. París: Autor
Universidad de Chile, Santiago, Programa de Estudios Sistémicos de Envejecimiento y Vejez, (2009, abril). Inclusión y exclusión social del adulto mayor en Chile. Recuperado el 2 de julio de 2009, del sitio web del Ministerio Secretaría General de la Presidencia: [Documento en línea] Disponible:

http://www.senama.cl/dinamico/noticias. aspx?idTd=9\&idDo=2967 [Consulta: 2012, Noviembre 12]. 